\title{
Different Types of Radio Sources and Possible Evolution of Radio Galaxies
}

\author{
Gabriel A. Ohanian \\ Byurakan Astrophysical Observatory, 378433, Byurakan, Aragatzotn \\ province, Armenia
}

Extragalactic radio sources have been studied for many years, but it is still unclear how they are formed and evolve. The sizes of the most powerful radio emitters in the Universe vary from less than one parsec to more than $1 \mathrm{Mpc}$. This large range of sizes has been interpreted as evidence for the evolution of the linear sizes of radio structure (e.g., O'Dea and Baum, 1997). A crucial element in the study of their evolution is the identification of the young compact counterparts of "old" FRI/FRII extended objects. Good candidates for young radio sources are those with peaked spectra (Gigahertz Peaked Spectrum - GPS and Compact Steep Spectrum - CSS, e.g., O'Dea 1998). Radio sources are presumably born in the very compact GPS phase, then they expand beyond $1 \mathrm{kpc}$ into the CSS regime and finally, they reach a size of $20 \mathrm{kpc}$, and afterwards evolve into large-scale radio sources (young scenario, e.g., O'Dea 1998). Alternatively, GPS sources may be compact because a particularly dense environment prevents them from growing larger (old scenario, e.g., O'Dea 1998). In either scenario, the radio source host galaxy determines the time evolution of the radio structure. By studying the optical environments and host galaxies we hope to obtain clues to the evolution of the radio sources. Similarities or differences in host galaxy properties over a range of radio source types and sizes enable us to investigate possible differences or similarities of the radio size class as a whole.

It is hence of crucial importance to enlarge a multispectral sample of different classes of radio sources. Proceeding from this position we have formed GPS, CSS and VSS (Very Steep Spectrum) radio source samples selected from the Ooty lunar occultation survey at $327 \mathrm{MHz}$, which have flux limits about 30 times fainter than that of the $3 \mathrm{C}$ (Singal 1987, and references therein). About 300 radio sources from the Ooty lists have been observed by us at $102 \mathrm{MHz}$ on the Large Phased Array (Pushchino, Russia) and on the RATAN-600 radiotelescope (Zelenchukskaya, Russia) at 968, 2300, 3950 and $7700 \mathrm{MHz}$. Details of these observations, the spectra and the list of radio sources are presented by Ohanian, 1998. Of these observed sources, 232 have spectra determined by 4 or more flux density points, at different frequencies. From those 232 classified spectra we select the above mentioned samples of radio sources.

In this poster we present a comparison of the 3 samples properties and a preliminary qualitative discussion. We have compared the properties of the VSS, CSS and GPS classes of radio sources in Table 1.

If the steepness of the optically-thin part of the radio spectrum in CSS and GPS sources is caused by radiative and adiabatic losses, then the break in the spectrum will move to lower frequencies as time increases (Kardashev 1962). The steepness of the spectra in GPS sources may be evidence for inverse Compton losses, which are significant in the early epoch of evolution of radio 
Table 1. Some parameters of different samples of radio sources

\begin{tabular}{ccccc}
\hline $\begin{array}{c}\text { Samples } \\
\text { (number } \\
\text { of r.s.) }\end{array}$ & $\begin{array}{c}\text { Mean } \\
\text { spec. } \\
\text { index. }\end{array}$ & $\begin{array}{c}\text { Relative } \\
\text { number } \\
\text { of gal. }\end{array}$ & $\begin{array}{c}\text { Relative } \\
\text { number } \\
\text { of EF r.s. }\end{array}$ & $\begin{array}{c}\text { Relative } \\
\text { number } \\
\text { of QSO }\end{array}$ \\
\hline GPS(26) & 1.25 & $20 \%$ & $48 \%$ & $32 \%$ \\
CSS(47) & 1.06 & $32 \%$ & $51 \%$ & $17 \%$ \\
VSS(48) & 1.11 & $20 \%$ & $67 \%$ & $13 \%$ \\
\hline
\end{tabular}

sources (Kardashev 1962). As we see from Table 1 the mean spectral indices of the VSS and CSS sources are similar. This corresponds to Kardashev's assumption, that in time the steepness of the spectrum stays constant or rises. That is, energy losses are offset by relativistic electron injection. The injection of relativistic electrons takes place by continuous and/or by recurrent bursts. From the point of view of V. A. Ambartsumian (1968), bursts which are accompanied by high energy release take place in galaxies where the core luminosity is not high (in this case radio luminosity, i.e. radio-quiet objects), and more quiet forms of activity are associated with bright cores (in this case - radio loud objects). Further, in the last decade several studies have shown that variability is anti-correlated with luminosity, in the sense that luminous QSOs have smaller amplitude variations than low luminosity ones (e.g. Hook et al., 1994). For three elliptical galaxies - NGC 1052, NGC 5077 (core is GPS type), and IC 1459 - that have been observed for at least $20 \mathrm{yr}$., it is noticed that the radio emission varies monotonically on time-scales of decades, though bursts in NGC 1052 and 5077 clearly occur on much shorter timescales (Slee et al., 1994). However, in radio-quiet objects monotonic variations are not typical. It is possible that in the radio-quiet objects, re-supply of relativistic electrons takes place only by separate bursts, i.e., the continuum injection component is negligible. Hence, in these objects we do not observe large-scale radio structure. In radio-loud objects, the presence of large-scale radio structures implies that a continuous resupply of electrons must take place. Superposed on them could be a burst component. That is, if a radio-loud object exists in a dense interstellar medium (like Seyfert type galaxies), then the medium cannot impede the outward propagation of the radio source, due to the continuous resupply of sufficient energy to the radio source structure.

If it's true that the GPS and CSS sources are young versions of the large radio sources, it means that large radio sources in host galaxies would pass through the GPS and CSS phase. The maximum lifetime of radio sources is $10^{7.5}$ years (Alexander and Leahy, 1987). In that time the host galaxies of large scale radio sources must live through a GPS-CSS-VSS evolutionary sequence. As we see from Table 1, the relative number of QSOs continually decreases when passing through the GPS-CSS-VSS sequence, and consequently, at that time the relative number of galaxies plus EFs (EFs are extremely faint $-\mathrm{m}>21$ galaxies) are increasing at the same rate. It means that QSOs are changed to the galaxies. Thus, if it's true that the radio source evolutionary sequence is a transition from the GPS phase to the CSS phase and then to the VSS phase, then Table 1 is the possible evolutionary scheme of radio galaxies. 


\section{References}

Alexander P. \& Leahy J. P. 1987, MNRAS, 225, 1

Ambartsumian, V. A. 1968, IAU Symp. 29, 11

de Vries W. H., O'Dea C.P., Baum S. A., Perlman E., Lehnert M. D. \& Barthel P. D. 1998, STSI prep. No 1226,1

Hook J. M., McMahon R. G., Boyle B. J. \& Irwin M. J. 1994 MNRAS, 268, 305

O'Dea, C. P. and Baum, S. A. 1997, AJ, 113, 148

O'Dea, C. P. 1998, STSI prep. No 1216

Ohanian G. A. 1997, PhD Thesis, Byurakan

Kardashev, N. S. 1962, Soviet Ast., 6, 317

Singal, A. K. 1987, A\&AS, 69, 91

Slee, O.B., Sadler Elaine M., Reynolds J. E. \& Ekers R. D. 1994, MNRAS, 269, 928 